\title{
Comparative analysis of mRNA transcripts of HT-29 cell line expressed in identical quantities for pathogenic $E$. coli strains UM146 and UM147 with control Escherichia coli Nissle 1917
}

\author{
Roman Kotłowski ${ }^{凶}$ \\ Department of Molecular Biotechnology and Microbiology, Gdansk University of Technology, Faculty of Chemistry, Narutowicza 11/12, 80-233 Gdansk, \\ Poland. \\ $\triangle$ Corresponding author: Department of Molecular Biotechnology and Microbiology, Gdansk University of Technology, Faculty of Chemistry, Narutowicza \\ 11/12, 80-233 Gdansk, Poland; Tel.: +48583472383, Fax: +48583471822; Email: romkotlo@pg.edu.pl. \\ (c) The author(s). This is an open access article distributed under the terms of the Creative Commons Attribution License (https://creativecommons.org/licenses/by/4.0/). \\ See http:/ /ivyspring.com/terms for full terms and conditions.
}

Received: 2021.09.19; Accepted: 2021.11.17; Published: 2022.01.01

\begin{abstract}
Aim of study was comparative analysis of mRNA transcripts of HT-29 cell line, expressed in identical quantities for the combination of pathogenic and non-pathogenic Escherichia coli strains. HT-29 confluent monolayers infection with two pathogenic $E$. coli strains UM146 and UM147 resulted in two sets of mRNA transcripts that were identical with RNA transcripts obtained for non-pathogenic one strain $E$. coli Nissle 1917. In this study genome-wide experiments were conducted using expression microarray-system. Only one common mRNA transcript coding for CCDC65 gene was equally expressed by HT-29 cells after incubation challenge with three different $E$. coli strains used. This gene and its bacterial analogue are important in the ciliary or flagellar motility, respectively. Altogether, 78 and 81 HT-29 mRNA transcripts for E. coli UM146 and E. coli UM147 had identical RNA quantity in comparison to the response obtained for non-pathogenic E. coli Nissle 1917 interactions with HT-29 monolayers. Specific analysis using REACTOME and agriGO terms enrichment data-mining tools as well as word-cloud analysis allowed for identification the most important processes characteristic during HT-29 cell line infections for each pathogenic $E$. coli strain used. The importance of results may contribute to recognition of those processes during bacterial infections that are identical with processes arising from human interaction with non-pathogenic strains that belong to the same bacterial species.
\end{abstract}

Key words: HT-29 cell line; Escherichia coli; PAI I; trypsin-like activity; infection

\section{Introduction}

The majority of studies concerning hostpathogen interactions are focused on characterization of the pathogenic bacterial strains in confrontation to non-pathogenic control bacterial species. However, in this study different strategy during subtractive experiment in the human-genome wide scale was conducted. The difference was related to identification of only those mRNA transcripts that were expressed in the same quantity also for non-pathogenic bacterial strain used as control. The obtained results of our model studies may help to understand the basis of immunological responses that are constant regardless of invading or existing in commensal manner strains that interact with host cells. Also, some implications can be made for understanding the mechanisms of human infections from variety of bacterial toxins present in two different $E$. coli strains and different $H$ flagellar types and some minor differences in amino-acid sequences of FimH, Mdh and PurA proteins previously reported [1].

E. coli isolated from Crohn's Disease patient and E. coli strain derived from Ulcerative Colitis patient used in this host-pathogen model study extend the 
current knowledge about inhibitory effect of Escherichia coli strain Nissle 1917 (EcN) on adhesion to and invasion of intestinal epithelial cells during probiotic treatment with EcN [2]. Also, Lactobacillus casei DN-114001 [3] known by strong inhibitory interaction of adherent-invasive E. coli with intestinal epithelial cells confirms the significant role of presented studies in better understanding of bacterial aetiology of Crohn's Disease.

\section{Materials and methods}

\section{Cell line}

HT-29 cell line stored originally in liquid nitrogen was cultured in the RPMI 1640 medium supplemented in $10 \%$ with fetal bovine serum until $70 \%$ of the bottom line of culture bottles with the surface of $75 \mathrm{~cm}^{2}$ were covered by HT-29 cells monolayer. Observation by inverted microscope was conducted to estimate the confluent status of cell line. Subsequently, 3h incubation time of $1 \mathrm{ml}$ bacterial cultures $\approx 10^{7} \mathrm{CFU}$ followed by pipetting of confluent cell line was conducted at $37^{\circ} \mathrm{C}$ at the presence of normal atmosphere supplemented with $5 \% \mathrm{CO}_{2}$.

\section{Micro-array}

The total RNA was extracted from HT-29 cell line based on Chmczynski and Sacchi protocol following further RNA processing in accordance with the Affymetrix ${ }^{\circledR}$ recommendations for GeneChip HG-U133A Plus 2.0 microarray expression chips protocol. Subsequently, biotinylated RNA was stained in fluidic station and emission of signals for entire human genome was determined using Affymetrix ${ }^{\circledR}$ scanner.

\section{Bacterial strains}

Pathogenic E. coli strains UM147 and UM146 have differences identified in amino-acid sequence of Mdh coding for malate dehydrogenase, PurA encoding adenylosuccinate dehydrogenase and FimH protein present in fimbria type I. In addition, E. coli UM146 has PAI I pathogenicity island and has H7 type of flagella in contrast to H5-type present in UM147 and H1 present in control strain E. coli Nissle 1917. However, unique feature of E. coli UM147 is trypsin like activity determined during cell line culture.

\section{Statistical analysis}

Statistical test methods applied in agriGO: GO Analysis Toolkit and Database for Agricultural Community (cau.edu.cn) (http://bioinfo.cau.edu.cn/ agriGO/) were as follows: Chi-Square; Multi-test adjustment method Hohberg FDR; Significant level 0.1. In the REACTOME online tool only biochemical reactions that significantly $(\mathrm{p}<0.05)$ represented particular biochemical pathway were selected for comparative analysis.

\section{Results and Discussion}

In the present study two sets of RNA transcripts determined for HT-29 genes after infection with two pathogenic E. coli strains UM146 and UM147 and one non-pathogenic E. coli Nissle 1917. The subtraction criteria relayed on identical quantity of cell-line transcripts response for two separately analyzed pathogenic E. coli strains with non-pathogenic one. Since, as it was found an E. coli UM146 was an intracellular strain in confrontation with the cell line and E. coli UM147 was an example of attaching to the HT-29 cells variant type pathogen, different pools of genes were expressed after $3 \mathrm{~h}$ incubation time for these two pathogenic strains. Only one common gene transcript CCDC65 with identical RNA quantity in HT-29 cells after incubation with three E. coli strains tested, was identified (Table 1). CCDC65 plays a critical role in ciliary and flagellar motility driven by the nexin-dynein regulatory complex (N-DRC) as it has been demonstrated using defective gene mutations study by Bower et al. [4]. REACTOME analysis revealed that only 6 RNA transcripts among 78 identified participated in 16 biochemical pathways for E. coli UM146 treated monolayer adenocarcinoma cells. In the case of E. coli UM147 16 RNA transcripts among 81 detected were involved in 50 biochemical pathways presented in Table 2A. Platelet aggregation initiated by E. coli UM146 suggests risk of heart failure in the case of human infection. Proteins involved in this process RAP1A and ITGB3 participate in 24 out of 27 possible biochemical reactions of plug formation known in REACTOME database. In addition, the same two proteins RAP1A and ITGB3 are involved in all 24 possible bioreactions during the response of HT-29 monolayer cells to bacterial invasion stimulated by integrins (Table 2A). Phospholipid metabolism regulated by PIKFYVE, SLC44A2, MTMR14 and HADH proteins was initiated in concordance with beta-oxidation processes of phospholipids fatty acids after incubation of HT-29 adenocarcinoma cells with E. coli UM147 (Table 2B). Also, several interleukins including interleukins 2, 3, $5,7,9,15,21$ involved in signaling processes of plausible infection progress were activated during this bacterial interaction with HT-29 cells, as indicated in Table 2B. In addition, autophagy process was initiated by E. coli UM147 as indicated by expression of mRNA transcripts for RAB40B, ATG16L2, MTMR14 and MLST8 proteins what means that phagocytosis process was initiated by HT-29 cells. 
$\mathrm{Y}=\mathrm{p}$-value $\leftarrow$ direction of numbering from 1 to 9 or 13 like in Table 3

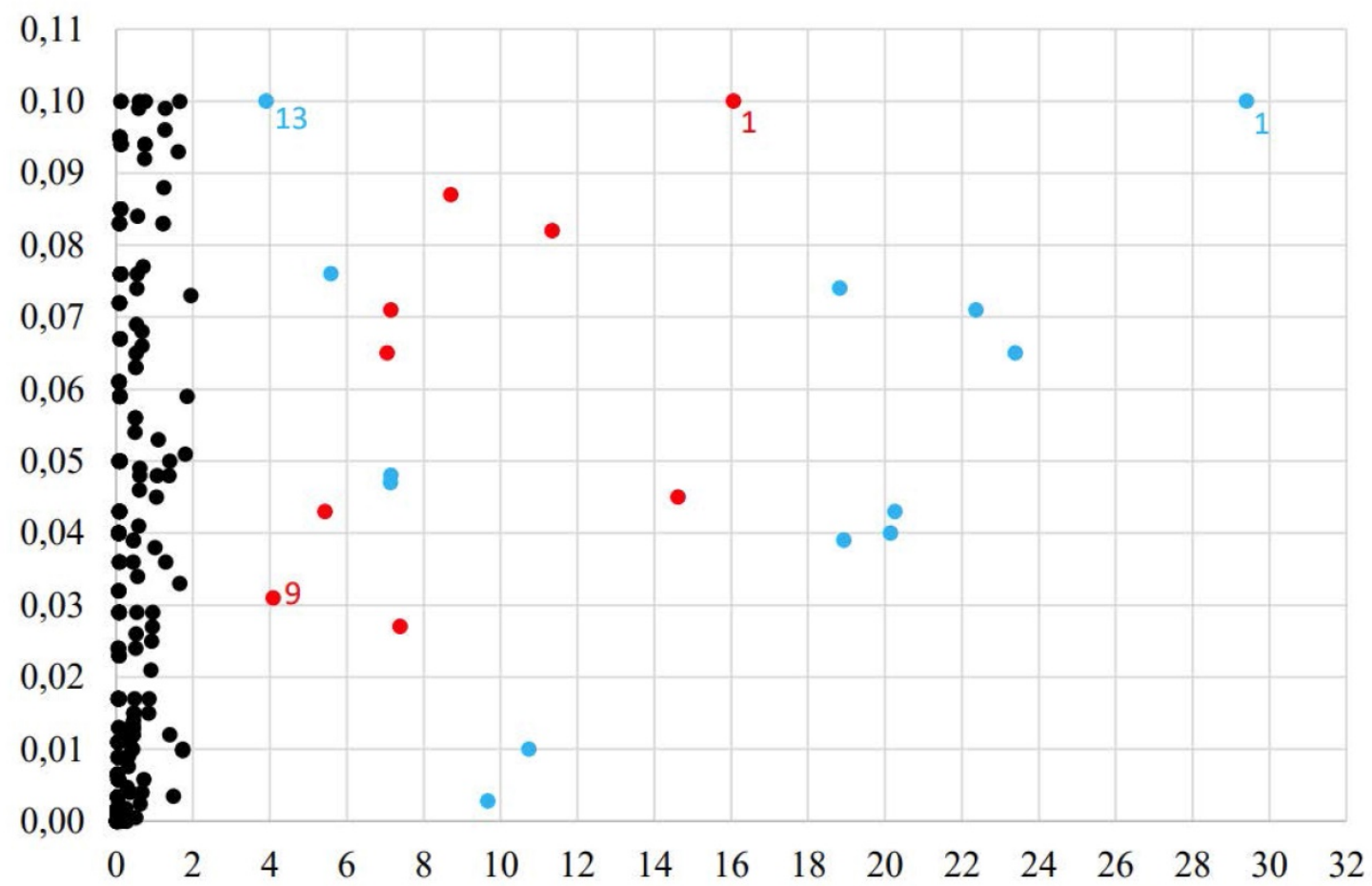

$\mathrm{X}=$ is the $\%$ ratio between observed and total number of the Gene Ontology identical or very similar terms in the entire Homo sapiens genome

Figure 1. The most relevant Gene Ontology terms including Processes, Functions and Cellular Component in the response to E. coli UM146 (red spots) and E. coli UM147 (blue spots).

Table 1. Equal HT-29 cell line RNA transcripts expression after $3 \mathrm{~h}$ incubation with $E$. coli strains used

\begin{tabular}{ll}
\hline E. coli strains & RNA transcripts \\
\hline E. coli UM146 and E. coli Nissle & ARV1, ATXN3L, ATXN7L1, C10orf126, C11orf31, C18orf54, C20orf196, C21orf58, CCDC65, CEP162, CH507-42P11.6, DDC-AS1, DDX10, \\
1917, n=78 & ECHDC3, FAM105A, FAM208B, FERMT2, FHL1, FOXP1, FRMD7, GALR3, H2BFXP, HBE1, ITGB3, JPH1, LINC00319, LINC00485, \\
& LOC100101478, LOC100287015, LOC101927268, LOC101928837, LOC102724851, LOC105374994, LOC286178, LOC440982, LOC613266, \\
& LOC643733, MICU1, MSRB3, NAA30, NCOA2, NCR1, NFAM1, NR2F1-AS1, NR3C1, NUDT17, NXPE3, OR52K3P, OVOL2, PBX4, \\
& PCDH12, PCDHB18P, PGM2L1, PGR, PIEZO1, PPP1R3C, PRELP, PWP2, RALGAPA2, RAP1A, REC8, S100G, SAMSN1, SERPINB1, \\
& SEZ6L, SLCO3A1, SPERT, SRSF1, SSFA2, THADA, TLE4, TNXB, UBXN8, UNC5C, WIPI2, ZEB2, ZIC1, ZNF775 \\
E. coli UM147 and E. coli Nissle & AGFG2, AGRN, ATG16L2, BATF2, C4orf47, CALB2, CALML3, CCDC65, CDRT1, CEACAM7, DECR2, E4F1, EHF, ENTPD1, ETV1, F11, \\
1917, n=81 & FGF18, FMNL1, FOXA1, GPATCH4, GYG2, HADH, IFT140, IGHD3-16, LCP2, LGALS8, LINC00032, LOC100505841, LOC101927027, \\
& LOC101928020, LOC101928433, LOC105372733, LOC1720, LOXL2, MAPK8IP3, MARK1, MBTPS2, MED23, MLST8, MPEG1, MTMR14, \\
& MYO19, MYO3A, MYO6, NPHP4, NUP214, OGFRL1, OPLAH, PCDHAC2, PEBP1, PIAS1, PIKFYVE, PMPCA, POU2F3, PYGB, RAB40B, \\
& RALGDS, RHBDL1, RIPPLY3, RORC, RP9P, RTP3, SERBP1, SHCBP1L, SLC18A2, SLC1A2, SLC44A2, SMARCA4, SPAG8, STAT5A, \\
& THAP4, TLK1, TNP2, TOR3A, VEPH1, XPNPEP2, YY2, ZC3H7B, ZFP36L1, ZNF117, ZNF491
\end{tabular}

During the second data-mining analysis by agriGO online tool very intriguing negative regulation of adaptive immune response process was determined in the case of E. coli UM147 strain presented in Table 3 and Figure 1. Obtained result is an example of more precise in our opinion characterization of signaling interleukin processes of infection progress caused by this particular pathogenic strain in comparison to the results obtained from previous analysis. Furthermore, neural crest cell development process was also enriched in this analysis. What is interesting, the neural crest is a collection of multipotent stem cells that helps to develop the autonomic nervous system and is characterised by multipotency providing possible future applications in regenerative medicine [5]. In the case of other pathogenic E. coli strain only general processes like positive regulation of transcription from RNA polymerase II promoter and positive regulation of RNA metabolic process were highlighted. Also, morphogenesis processes involved in HT-29 cell differentiation were significantly enriched $(\mathrm{p}<0.05)$ for E. coli UM146 strain (Table 3). 


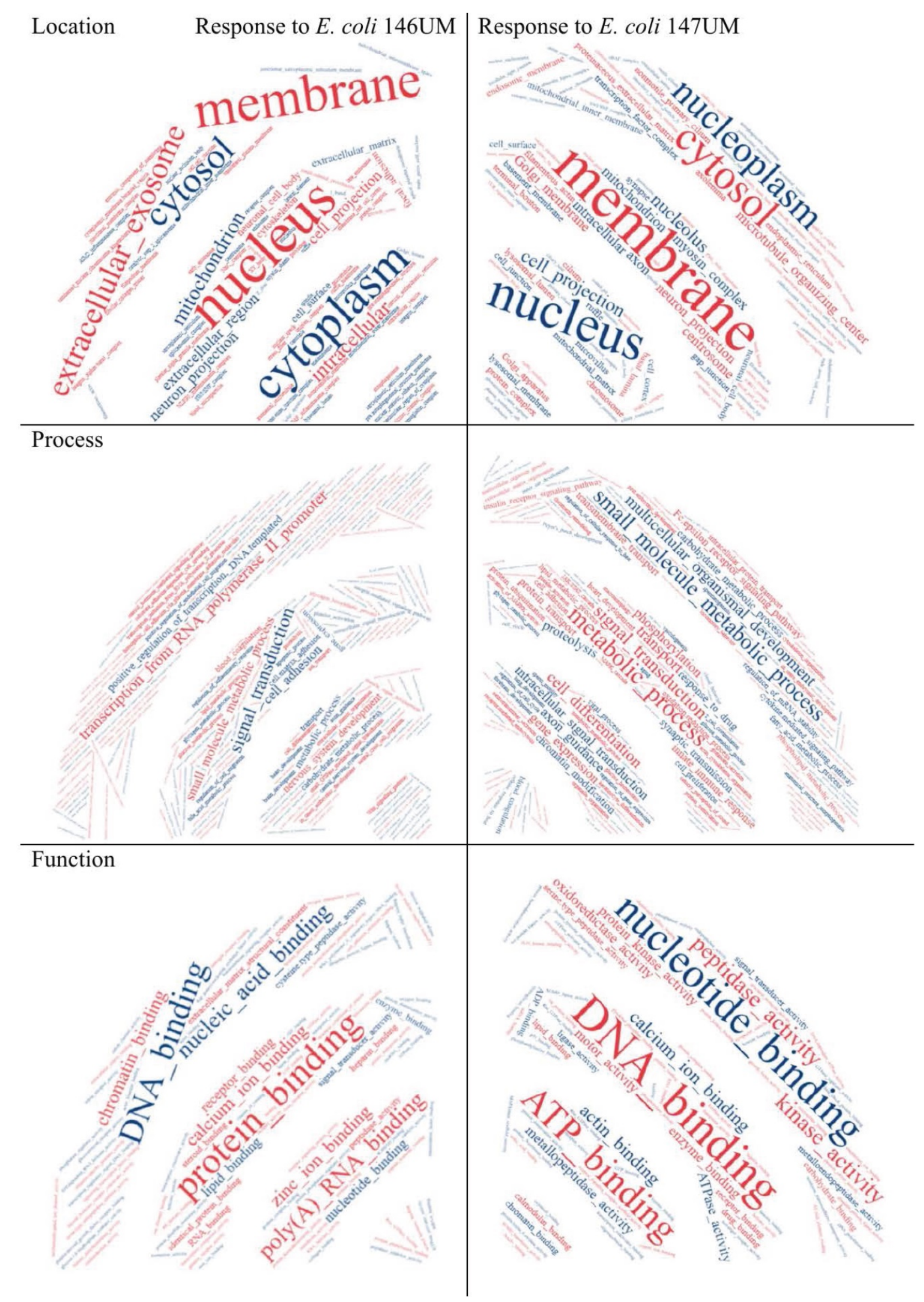

Figure 2. Ontology terms highlighted in word cloud application (https://www.wordclouds.com/) for all significant $(p<0.1)$ results obtained from agriGO search. 
Word cloud application results presented in Figure 2 provide general point of view on Gene Ontology terms predominantly enriched for Cellular Components what means location in HT-29 cells, Processes and Functions. Extracellular exosome was highlighted in location analysis what can be explained by trypsin-like activity of E. coli UM147 strain that is able to degrade intercellular connections in HT-29 monolayers. Some similarity in mode of action against Eucaryotic cells, can be seen in comparison to BFT toxins produced by enterotoxigenic Bacteroides fragilis strains [6], resulting in possible inflammation of the GI-tract [7, 8]. Transcription from RNA polymerase II promoter was highlighted among Processes for E. coli UM146 what is in agreement with the enrichment analysis from agriGO online tool applied. In the case of Functions, the most divergent parameter in the word-cloud analysis performed, peptidase and metallopeptidase activities were highlighted for E. coli UM147 what confirms previous conclusions about influence of trypsin-like activity on possible inflammation of the gastrointestinal tract in some IBD patients. Also, kinase activity was indicated as characteristic form E. coli UM 147 strain infection, what can suggest more active involvement of phosphorylation and/or dephosphorylation processes during protein-to-protein signalling regulation processes inside infected monolayer cells. For E. coli UM146 characteristic highlighted functions were however related to chromatin and poly(A) RNA molecules binding as well as calcium and zinc ion binding interactions usually occurring in the catalytic centers of enzymatic reactions more frequent in HT-29 cells during the course of UM146 E. coli infection model used.

Table 2. REACTOME Significant $(p<0.05)$ pathways detected in HT-29 cell line in the response to E. coli 146UM or E. coli 147UM infections identical with the control non-pathogenic $E$. coli Nissle 1917

\begin{tabular}{|c|c|c|c|c|c|}
\hline Pathway identifier & Pathway name & Entities pValue & \#Reactions found & \#Reactions total & Mapped entities \\
\hline \multicolumn{6}{|c|}{ A. Response to E. coli UM146 } \\
\hline R-HSA-383280 & Nuclear Receptor transcription pathway & 1,071743E-11 & 2 & 2 & PGR;NR3C1 \\
\hline R-HSA-354194 & GRB2: SOS provides linkage to MAPK signaling for Integrins & $7,293580 \mathrm{E}-03$ & 2 & 2 & RAP1A;ITGB3 \\
\hline R-HSA-372708 & p130Cas linkage to MAPK signaling for integrins & $8,753419 \mathrm{E}-03$ & 3 & 3 & RAP1A;ITGB3 \\
\hline R-HSA-3322077 & Glycogen synthesis & 1,202797E-02 & 11 & 24 & PPP1R3C;PGM2L1 \\
\hline R-HSA-354192 & Integrin signaling & 2,567075E-02 & 24 & 24 & RAP1A;ITGB3 \\
\hline R-HSA-4090294 & SUMOylation of intracellular receptors & 2,689497E-02 & 2 & 25 & PGR;NR3C1 \\
\hline R-HSA-8982491 & Glycogen metabolism & $3,070566 \mathrm{E}-02$ & 12 & 39 & PPP1R3C;PGM2L1 \\
\hline R-HSA-6802948 & Signaling by high-kinase activity BRAF mutants & 3,202079E-02 & 4 & 6 & RAP1A;ITGB3 \\
\hline R-HSA-1251985 & Nuclear signaling by ERBB4 & 3,609635E-02 & 2 & 34 & PGR \\
\hline R-HSA-5674135 & MAP2K and MAPK activation & 3,891867E-02 & 8 & 12 & RAP1A;ITGB3 \\
\hline R-HSA-9656223 & Signaling by RAF1 mutants & 3,891867E-02 & 4 & 7 & RAP1A;ITGB3 \\
\hline R-HSA-76009 & Platelet Aggregation (Plug Formation) & 4,480482E-02 & 24 & 27 & RAP1A;ITGB3 \\
\hline R-HSA-6802955 & Paradoxical activation of RAF signaling by kinase inactive BRAF & 4,632490E-02 & 4 & 7 & RAP1A;ITGB3 \\
\hline R-HSA-9649948 & Signaling downstream of RAS mutants & 4,632490E-02 & 4 & 7 & RAP1A;ITGB3 \\
\hline R-HSA-6802946 & Signaling by moderate kinase activity BRAF mutants & 4,632490E-02 & 4 & 7 & RAP1A;ITGB3 \\
\hline R-HSA-6802949 & Signaling by RAS mutants & 4,632490E-02 & 4 & 9 & RAP1A;ITGB3 \\
\hline \multicolumn{6}{|c|}{ B. Response to E. coli UM147 } \\
\hline R-HSA-70221 & Glycogen breakdown (glycogenolysis) & $6,172902 \mathrm{E}-04$ & 12 & 15 & PYGB;GYG2 \\
\hline R-HSA-1266695 & Interleukin-7 signaling & 1,022987E-03 & 10 & 26 & STAT5A;SMARCA4 \\
\hline R-HSA-9645135 & STAT5 Activation & $1,512084 \mathrm{E}-03$ & 3 & 3 & STAT5A \\
\hline R-HSA-9027283 & Erythropoietin activates STAT5 & $1,512084 \mathrm{E}-03$ & 3 & 3 & STAT5A \\
\hline R-HSA-8949275 & RUNX3 Regulates Immune Response and Cell Migration & $1,859202 \mathrm{E}-03$ & 2 & 5 & RORC \\
\hline R-HSA-8985947 & Interleukin-9 signaling & 2,240519E-03 & 4 & 13 & STAT5A \\
\hline R-HSA-8982491 & Glycogen metabolism & $2,586785 \mathrm{E}-03$ & 21 & 39 & PYGB;GYG2 \\
\hline R-HSA-9020958 & Interleukin-21 signaling & 2,655603E-03 & 2 & 5 & STAT5A \\
\hline R-HSA-2586552 & Signaling by Leptin & 3,104028E-03 & 5 & 19 & STAT5A \\
\hline R-HSA-9020558 & Interleukin-2 signaling & $3,585370 \mathrm{E}-03$ & 5 & 19 & STAT5A \\
\hline R-HSA-8983432 & Interleukin-15 signaling & 4,645137E-03 & 5 & 17 & STAT5A \\
\hline R-HSA-77350 & Beta oxidation of hexanoyl-CoA to butanoyl-CoA & 5,831597E-03 & 2 & 4 & $\mathrm{HADH}$ \\
\hline R-HSA-77310 & Beta oxidation of lauroyl-CoA to decanoyl-CoA-CoA & 5,831597E-03 & 2 & 4 & HADH \\
\hline R-HSA-77348 & Beta oxidation of octanoyl-CoA to hexanoyl-CoA & $5,831597 \mathrm{E}-03$ & 2 & 4 & $\mathrm{HADH}$ \\
\hline R-HSA-1170546 & Prolactin receptor signaling & 5,831597E-03 & 2 & 14 & STAT5A \\
\hline R-HSA-9702518 & STAT5 activation downstream of FLT3 ITD mutants & 7,841754E-03 & 13 & 14 & STAT5A \\
\hline R-HSA-77346 & Beta oxidation of decanoyl-CoA to octanoyl-CoA-CoA & 7,841754E-03 & 2 & 5 & HADH \\
\hline R-HSA-1839117 & Signaling by cytosolic FGFR1 fusion mutants & 9,330878E-03 & 1 & 14 & STAT5A \\
\hline R-HSA-2243919 & Crosslinking of collagen fibrils & 1,011898E-02 & 3 & 13 & LOXL2 \\
\hline R-HSA-9725371 & Nuclear events stimulated by ALK signaling in cancer & $1,265287 \mathrm{E}-02$ & 2 & 9 & STAT5A \\
\hline R-HSA-9670439 & $\begin{array}{l}\text { Signaling by phosphorylated juxtamembrane; extracellular } \\
\text { and kinase domain KIT mutants }\end{array}$ & 1,355277E-02 & 4 & 11 & STAT5A \\
\hline R-HSA-9669938 & Signaling by KIT in disease & 1,355277E-02 & 4 & 25 & STAT5A \\
\hline R-HSA-982772 & Growth hormone receptor signaling & 1,447970E-02 & 5 & 28 & STAT5A \\
\hline R-HSA-8854691 & Interleukin-20 family signaling & 1,447970E-02 & 3 & 56 & STAT5A \\
\hline R-HSA-1632852 & Macroautophagy & 1,488191E-02 & 10 & 87 & RAB40B;ATG16L2; \\
\hline
\end{tabular}




\begin{tabular}{|c|c|c|c|c|c|}
\hline Pathway identifier & Pathway name & Entities pValue & \#Reactions found & \#Reactions total & Mapped entities \\
\hline & & & & & MTMR14;MLST8 \\
\hline R-HSA-1226099 & Signaling by FGFR in disease & $1,507210 \mathrm{E}-02$ & 27 & 106 & STAT5A;FGF18 \\
\hline R-HSA-9703648 & Signaling by FLT3 ITD and TKD mutants & 1,543329E-02 & 13 & 24 & STAT5A \\
\hline R-HSA-9703465 & Signaling by FLT3 fusion proteins & $1,741900 \mathrm{E}-02$ & 8 & 18 & STAT5A \\
\hline R-HSA-9674555 & Signaling by CSF3 (G-CSF) & 2,058859E-02 & 9 & 21 & STAT5A \\
\hline R-HSA-9006335 & Signaling by Erythropoietin & $2,058859 \mathrm{E}-02$ & 3 & 24 & STAT5A \\
\hline R-HSA-9612973 & Autophagy & 2,071701E-02 & 10 & 108 & $\begin{array}{l}\text { RAB40B;ATG16L2; } \\
\text { MTMR14;MLST8 }\end{array}$ \\
\hline R-HSA-186763 & Downstream signal transduction & 2,282495E-02 & 1 & 16 & STAT5A \\
\hline R-HSA-5578998 & Defective OPLAH causes OPLAHD & 2,472363E-02 & 1 & 1 & OPLAH \\
\hline R-HSA-3878781 & Glycogen storage disease type IV (GBE1) & 2,472363E-02 & 1 & 1 & GYG2 \\
\hline R-HSA-3858516 & Glycogen storage disease type 0 (liver GYS2) & 2,472363E-02 & 1 & 1 & GYG2 \\
\hline R-HSA-1839124 & FGFR1 mutant receptor activation & 2,515676E-02 & 1 & 25 & STAT5A \\
\hline R-HSA-3232142 & SUMOylation of ubiquitinylation proteins & 3,009615E-02 & 2 & 3 & NUP214;PIAS1 \\
\hline R-HSA-9682385 & FLT3 signaling in disease & 3,009615E-02 & 15 & 52 & STAT5A \\
\hline R-HSA-3249367 & STAT6-mediated induction of chemokines & 3,080947E-02 & 3 & 6 & STAT5A \\
\hline R-HSA-1566948 & Elastic fibre formation & $3,403180 \mathrm{E}-02$ & 1 & 17 & LOXL2 \\
\hline R-HSA-451927 & Interleukin-2 family signaling & 3,538602E-02 & 16 & 59 & STAT5A \\
\hline R-HSA-9607240 & FLT3 Signaling & $3,676091 \mathrm{E}-02$ & 3 & 43 & STAT5A \\
\hline R-HSA-5655302 & Signaling by FGFR1 in disease & $3,815614 \mathrm{E}-02$ & 1 & 35 & STAT5A \\
\hline R-HSA-512988 & Interleukin-3; Interleukin-5 and GM-CSF signaling & 3,957142E-02 & 5 & 38 & STAT5A \\
\hline R-HSA-1433557 & Signaling by SCF-KIT & $4,100645 \mathrm{E}-02$ & 4 & 39 & STAT5A \\
\hline R-HSA-9673221 & Defective F9 activation & $4,286870 \mathrm{E}-02$ & 1 & 1 & F11 \\
\hline R-HSA-77286 & mitochondrial fatty acid beta-oxidation of saturated fatty acids & $4,393460 \mathrm{E}-02$ & 15 & 29 & HADH \\
\hline R-HSA-1483191 & Synthesis of PC & $4,693824 \mathrm{E}-02$ & 1 & 18 & SLC44A2 \\
\hline R-HSA-1483257 & Phospholipid metabolism & $4,814536 \mathrm{E}-02$ & 11 & 218 & $\begin{array}{l}\text { PIKFYVE;SLC44A2; } \\
\text { MTMR14;HADH }\end{array}$ \\
\hline
\end{tabular}

1: Pathway identifier; 2: Pathway name; 3: Reactions found: The number of reactions in the pathway that are represented by at least one molecule in the submitted data set; 4: Reactions Total: The number of reactions in the specific pathway name that contain molecules found; 5 : Entities p-value: The result of the statistical test for over-representation of RNA transcripts in reactions identified; 6: Mapped entities: RNA transcripts participating in pathways.

Table 3. AgriGO comparison of HT-29 cell line transcription responses to pathogenic E. coli strains UM146 and UM147 equal to the level of transcription of non-pathogenic strain $E$. coli Nissle 1917

\begin{tabular}{|c|c|c|}
\hline No. & E. coli UM146 - red spots & E. coli UM147 - blue spots \\
\hline 1. & P: cellular component biogenesis & P: aminoglycan catabolic process \\
\hline 2. & P: cellular component assembly & P: regulation of bone remodeling \\
\hline 3. & P: nervous system development & $\begin{array}{l}\text { P: negative regulation of immune } \\
\text { system process }\end{array}$ \\
\hline 4. & $\begin{array}{l}\text { P: positive regulation of nucleobase, } \\
\text { nucleoside, nucleotide and nucleic acid } \\
\text { metabolic process }\end{array}$ & $\begin{array}{l}\text { P: regulation of phospholipase } \\
\text { activity }\end{array}$ \\
\hline 5. & $\begin{array}{l}\text { P: positive regulation of RNA metabolic } \\
\text { process }\end{array}$ & $\begin{array}{l}\text { P: embryonic organ } \\
\text { morphogenesis }\end{array}$ \\
\hline 6. & P: positive regulation of transcription & $\begin{array}{l}\text { P: positive regulation of } \\
\text { biosynthetic process }\end{array}$ \\
\hline 7. & $\begin{array}{l}\text { P: positive regulation of transcription, } \\
\text { DNA-dependent }\end{array}$ & P: amine metabolic process \\
\hline 8. & $\begin{array}{l}\text { P: positive regulation of transcription } \\
\text { from RNA polymerase II promoter }\end{array}$ & $\begin{array}{l}\text { P: negative regulation of adaptive } \\
\text { immune response }\end{array}$ \\
\hline 9. & $\begin{array}{l}\text { P: cell morphogenesis involved in } \\
\text { differentiation }\end{array}$ & P: neural crest cell development \\
\hline 10. & Less than 3 RNA transcripts involved & P: regulation of biological process \\
\hline 11. & & P: regulation of protein transport \\
\hline 12. & & P: oligodendrocyte differentiation \\
\hline 13. & & P: steroid biosynthetic process \\
\hline
\end{tabular}

From literature data about host-pathogen interactions using enteric bacteria we can highlight significant reduction of Campylobacter jejuni invasion and intracellular survival of HT-29 cell line after $4 \mathrm{~h}$ incubation period with $\mathrm{EcN}$ followed by infection with C. jejuni 81-176 [9]. The nature of EcN inhibitory interaction is by stimulating the expression of genes of cell junction and intestinal barrier integrity. As a result, there is an increase of the HT-29 cells resistance to infection. In our model of host-pathogen interaction between pathogenic and non-pathogenic bacterial strains and HT-29 cell line were separately studied based on identical RNA transcripts responses from the same pull of genes. From our model however it is possible to recognise biochemical reactions where probiotic and pathogenic strains equally participate in the same processes, what can give better insight into therapeutic effect during medical treatment.

\section{Competing Interests}

The authors have declared that no competing interest exists.

\section{References}

1. Sepehri S, Kotlowski R, Bernstein CN, et al. Phylogenetic analysis of inflammatory bowel disease associated Escherichia coli and the fimH virulence determinant. Inflamm Bowel Dis. 2009; 15:1737-45.

2. Boudeau J, Glasser AL, Julien S, Colombel JF, Darfeuille-Michaud A. Inhibitory effect of probiotic Escherichia coli strain Nissle 1917 on adhesion to and invasion of intestinal epithelial cells by adherentinvasive E. coli strains isolated from patients with Crohn's disease. Aliment Pharmacol Ther. 2003; 18:45-56.

3. Ingrassia I, Leplingard A, Darfeuille-Michaud A. Lactobacillus casei DN-114 001 inhibits the ability of adherent-invasive Escherichia coli isolated from Crohn's disease patients to adhere to and to invade intestinal epithelial cells. Appl Environ Microbiol. 2005; 71:2880-7.

4. Bower R, Tritschler D, Mills KV, et al. DRC2/CCDC65 is a central hub for assembly of the nexin-dynein regulatory complex and other regulators of ciliary and flagellar motility. Mol Biol Cell. 2018; 29:137-53.

5. Vega-Lopez GA, Cerrizuela S, Tribulo C, et al. Neurocristopathies: New insights 150 years after the neural crest discovery. Dev Biol. 2018; 444 Suppl. 1:S110-S143.

6. Wu S, Lim KC, Huang J, et al. Bacteroides fragilis enterotoxin cleaves the zonula adherens protein E-cadherin. Proc Natl Acad Sci USA. 1998; 95:14979-84. 
7. Kotlowski R, Bernstein $\mathrm{CN}$, Sepehri $\mathrm{S}$, et al. High prevalence of Escherichia coli belonging to the $\mathrm{B} 2+\mathrm{D}$ phylogenetic group in inflammatory bowel disease. Gut. 2007; 56:669-75.

8. Sepehri S, Kotlowski R, Bernstein $\mathrm{CN}$, et al. Microbial diversity of inflamed and noninflamed gut biopsy tissues in inflammatory bowel disease. Inflamm Bowel Dis. 2007; 13:675-83.

9. Helmy YA, Kassem II, Kumar A, Rajashekara G. In vitro evaluation of the impact of the probiotic E. coli Nissle 1917 on Campylobacter jejuni's invasion and intracellular survival in human colonic cells. Front Microbiol. 2017; 8:1588. 